\title{
Article
}

\section{Are low doses of caffeine as ergogenic as higher doses? A critical review highlighting the need for comparison with current best practice in caffeine research}

Pickering, Craig and Kiely, John

Available at http://clok.uclan.ac.uk/29558/

Pickering, Craig and Kiely, John ORCID: 0000-0001-9817-0224 (2019) Are low doses of caffeine as ergogenic as higher doses? A critical review highlighting the need for comparison with current best practice in caffeine research. Nutrition, 67-68 - p. 110535. ISSN 0899-9007

It is advisable to refer to the publisher's version if you intend to cite from the work. http://dx.doi.org/10.1016/j.nut.2019.06.016

For more information about UCLan's research in this area go to

http://www.uclan.ac.uk/researchgroups/ and search for <name of research Group>.

For information about Research generally at UCLan please go to http://www.uclan.ac.uk/research/

All outputs in CLoK are protected by Intellectual Property Rights law, including Copyright law. Copyright, IPR and Moral Rights for the works on this site are retained by the individual authors and/or other copyright owners. Terms and conditions for use of this material are defined in the policies page. 
2 Title: Are low doses of caffeine as ergogenic as higher doses? A critical review highlighting the need for

3 comparison to current best practice in caffeine research.

4

$5 \quad$ Short Title: Low versus high caffeine doses

6

7 Authors: Craig Pickering ${ }^{1}$, John Kiely ${ }^{1}$

8

9

1. Institute of Coaching and Performance, University of Central Lancashire, Preston, UK

10

11 Corresponding Author:

12 Craig Pickering

13 Institute of Coaching and Performance, University of Central Lancashire, Fylde Road, Preston, PR1 2HE, UK.

14 Email: craigpickering1014@ hotmail.com

15 Word Count: 4099

\section{Number of Tables: 1}

\section{Disclosure of potential conflicts of interest}

$18 \mathrm{CP}$ is a former employee of DNAFit LifeSciences, a genetic testing company. He received no financial incentives for the preparation of this manuscript. JK declares that he has no conflict of interest relevant to the content of this article.

21 


\section{$\underline{\text { Are low doses of caffeine as ergogenic as higher doses? A critical review highlighting the need for }}$}

\section{comparison to current best practice in caffeine research.}

\section{Abstract}

Caffeine is a popular and widely utilised sporting ergogenic aid. Over the years, the effects of different caffeine doses have been researched, with the general consensus being that 3-6 mg/kg of caffeine represents the optimal caffeine dose for most people. Recently there has been increased attention placed on lower ( $\leq 3 \mathrm{mg} / \mathrm{kg}) \mathrm{caffeine}$ doses, with some research suggesting these doses are also ergogenic. However, a critical consideration for athletes is not merely whether caffeine is ergogenic at a given dose, but whether the consumed dose provides an optimised performance benefit. Following this logic, we identify a potential oversight in the current research relating to the efficacy of lower caffeine doses. Although low caffeine doses do appear to bestow ergogenic effects, these effects have not been adequately compared to the currently accepted best practice dose of 3-6 $\mathrm{mg} / \mathrm{kg}$. This methodological oversight limits the practical conclusions we can extract from the research into the efficacy of lower doses of caffeine, as the relative ergogenic benefits between low and recommended doses remains unclear. Here, we examine existing research with a critical eye, and provide recommendations both for those looking to utilise caffeine to enhance their performance, and those conducting research into caffeine and sport.

Key Words: Caffeine, ergogenic, low-dose, supplement, sports drink 


\section{Introduction}

62

Of all sporting ergogenic aids, caffeine (1,3,7-trimethylxanthine) is the most popular, with approximately $75 \%$ of athletes consuming it either before or during competition [1,2]. Indeed, caffeine has such a reliable performance enhancing effect that, for over twenty years (1984-2004), high doses were banned for within-competition use by the World Anti-Doping Agency (WADA), and caffeine remains on their active monitoring programme to this day. The ergogenic effects of caffeine ingestion have been demonstrated across a wide range of sports, including endurance [3] and team sports [4], and across different exercise methods and modalities, including repeated high-intensity efforts [5], muscular endurance [6], maximum strength [7] and anaerobic performance [8].

Whilst the ergogenic effects of caffeine have been known for over 100 years [9], the broad array of potential mechanisms by which caffeine exerts its performance enhancing effects have only more recently been more fully elucidated. The most well-established mechanism is that of caffeine's role as a competitive adenosine receptor antagonist [10], dampening adenosine's downregulation of Central Nervous System arousal [11]. In turn, this promotes the release of a spectrum of neuro-chemicals, including dopamine and the excitatory neurotransmitter glutamate [12], thereby increasing muscle firing rates [13]. Caffeine also stimulates adrenaline secretion [14], alters substrate utilization and metabolism [15], and increases cellular ion release [16]. More recently, the relationship between caffeine, pain, and exercise performance has been explored, with current evidence suggesting that caffeine decreases pain perception, which in turn reduces rating of perceived exertion (RPE) [17] and enhances exercise capacity [18]. Latterly, it has been proposed that caffeine's bitter taste may drive some of its performance enhancing benefits [19], in a similar fashion to the documented effects of the bitter tasting compound quinine [20]; such observations may explain the ergogenic effects of caffeine-infused mouth-rinses [21].

Given that caffeine's effects have been extensively researched, and consistently, reliably and repeatedly demonstrated to improve — and only very rarely shown to harm [22]—exercise performance, it's use is pervasive amongst both professional and amateur athletes alike [1,2]. This extensive use has resulted in the 
89 formulation of best practice guidelines by numerous professional bodies. The International Society of Sports

90 Nutrition's position stand on caffeine [23], for example, summarizes that caffeine is effective at enhancing

91 performance at dosages considered to be moderate $(\sim 3-6 \mathrm{mg} / \mathrm{kg})$, consumed approximately 60 minutes prior to

92 performance, with no additional ergogenic effects seen with higher caffeine doses (>9 mg/kg). Such

93 recommendations have been echoed elsewhere, both in the scientific literature [14,16] and lay press.

94 Interestingly, however, a number of studies have recently shown that lower doses of caffeine, typically of $\leq 3$

$95 \mathrm{mg} / \mathrm{kg}$, are also ergogenic [24]. In this article, we examine the evidence underpinning this finding, and explore

96 whether low doses $(\leq 3 \mathrm{mg} / \mathrm{kg})$ of caffeine pre-exercise offer comparable ergogenic benefits to the more

97 conventionally recommended intakes $(3-6 \mathrm{mg} / \mathrm{kg})$; such an examination is crucial, as athletes are likely

98 interested in whether their caffeine dose offers the maximal ergogenic benefits, as opposed to just an ergogenic

99 effect. Finally, we note some methodological recommendations that researchers may wish to consider when

100 conducting low dose caffeine research in the future.

101

102

\section{Are low doses of caffeine ergogenic?}

103

104

Whilst, historically, high doses (up to $13 \mathrm{mg} / \mathrm{kg}$ ) of caffeine have been used to induce ergogenic effects

105 [25], more recently there has been an increasing focus on the use of more moderate ( $3-6 \mathrm{mg} / \mathrm{kg})$ caffeine doses

106 [26]. The success of these trials in turn has prompted research investigating the efficacy of lower doses of

107 caffeine $(\leq 3 \mathrm{mg} / \mathrm{kg})$. Whilst the number of these trials is relatively low, a recent review by Spriet [24] concluded

108 that these lower caffeine doses, when consumed prior to exercise, likely enhanced athletic performance.

109 Similarly, a recent meta-analysis of the ergogenic effects of caffeine-containing energy drinks, the majority of

110 which had a dose of $\leq 3 \mathrm{mg} / \mathrm{kg}$, concluded that ingestion of these drinks improved performance [27].

111 Accordingly, in general, the evidence to date supports the perspective that lower doses of caffeine are ergogenic

112 for sports performance, particularly with regards to endurance sport. However, perhaps a more pertinent

113 consideration for athletes is whether these low doses of caffeine are as effective in enhancing performance as

114 the more conventional, higher doses? As athletes consume caffeine primarily to improve performance, and

115 presumably wish to improve their performance to the maximum amount possible, this is an important

116 consideration. If low doses of caffeine are ergogenic, but not as ergogenic as higher doses, then athletes

117 consuming these lower doses may be leaving some potential performance improvements on the table. As such, 
118 the question as to whether or not low $(\leq 3 \mathrm{mg} / \mathrm{kg})$ doses of caffeine exert similar ergogenic effects as more

119 conventional, moderate (3-6 mg/kg) doses seems highly relevant.

120

There are two ways by which we could determine whether low doses of caffeine are as ergogenic as

122 higher doses. Firstly, we could compare the magnitude of improvements seen between studies; for example,

123 determining whether the size of the ergogenic effect is greater in those studies that utilise $6 \mathrm{mg} / \mathrm{kg}$ compared to

$1242 \mathrm{mg} / \mathrm{kg}$. This superficially simple approach, however, is surprisingly problematic, because the magnitude of

125 caffeine-derived performance enhancement is highly variable between both trials and subjects [28]. As

126 illustration, consider the array of variables which interact to modulate caffeine ergogenesis; genotype

127 [22,29,30], training status [31], habitual caffeine use [32], sex [33], caffeine source [34], age [35], expectancy

128 [36], exercise type [37], and time of day of exercise [38]. Given the extensive differences between study

129 methodologies and recruited populations, it seems unlikely that such a comparison would provide the desired,

130 and necessary, conceptual clarity.

131

132

Instead, a better option might be to have low-dose and high-dose caffeine trials within each study,

133 thereby allowing for a direct comparison between the different caffeine doses. Although seemingly sensible,

134 such an approach is surprisingly uncommon. In a recent review, Spriet [24] concluded that low caffeine doses

$135(\leq 3 \mathrm{mg} / \mathrm{kg})$, taken before exercise, enhanced athletic performance compared to placebo. However, the vast

136 majority of the studies included in Spriet's [24] review (summarized in table 1) did not directly compare a low

137 dose $(\leq 3 \mathrm{mg} / \mathrm{kg})$ of caffeine with a higher dose (>3 mg/kg). In fact, only 4 of the 14 studies did so [39-42]. Of

138 these four, there were mixed results; two reported no additional benefits from $6 \mathrm{mg} / \mathrm{kg}$ of caffeine compared to

$1393 \mathrm{mg} / \mathrm{kg}$ of caffeine when examining aerobic endurance performance $[39,41]$; one reported that $4.5 \mathrm{mg} / \mathrm{kg}$

140 enhanced aerobic endurance performance to a greater extent than $3.2 \mathrm{mg} / \mathrm{kg}$, which in turn was more ergogenic

141 than a dose of $2.1 \mathrm{mg} / \mathrm{kg}$ [40]; and one found that $5 \mathrm{mg} / \mathrm{kg}$ enhanced maximum knee flexion and extension

142 isokinetic torque, whilst $2 \mathrm{mg} / \mathrm{kg}$ did not [42]. The remaining studies either did not use a caffeine dose above 3

$143 \mathrm{mg} / \mathrm{kg}$ in their comparison [43-45], or only used a single caffeine dose ( $\leq 3 \mathrm{mg} / \mathrm{kg})$, and compared this to

144 placebo [46-52]. We identified additional papers published following Spriet's [24] review that directly

145 examined a low versus high dose of caffeine [22,53-56]. Of these, Arazi and colleagues [53] reported no

146 difference in performance between a low $(2 \mathrm{mg} / \mathrm{kg})$ and high $(5 \mathrm{mg} / \mathrm{kg})$ caffeine dose — a finding replicated by

147 Guest and colleagues [22] with doses of 2 and $4 \mathrm{mg} / \mathrm{kg}$ on a 10kg cycle ergometer time trial—whilst others [53- 
148 55] reported mixed results, in part because of the large number of performance tests utilised. Interestingly, Sabol

149 and colleagues [56] reported similar improvements in vertical jump performance following ingestion of 2, 4,

150 and $6 \mathrm{mg} / \mathrm{kg}$ of caffeine, whilst upper body ballistic exercise performance was only enhanced following a dose

151 of $6 \mathrm{mg} / \mathrm{kg}$. Consequently, due to both the equivocal results of the small numbers of trials directly investigating

152 this phenomenon, and the lack of higher caffeine doses utilised in other trials, it is unclear whether lower doses

153 of caffeine are as ergogenic as higher doses. Recently, Talanian \& Spriet [57] suggested that, based on their

154 interpretations of five lower-dose caffeine studies [26,40,43,44,57] that the timing of the lower caffeine dose may be a crucial aspect, with ingestion less than 60 minutes pre-exercise associated with a greater performance

156 benefit than later ingestion (80-180 minutes pre-exercise).

157

158

\begin{tabular}{|c|c|c|c|c|c|c|}
\hline Study & Subjects & $\begin{array}{l}\text { Caffeine } \\
\text { Timing }\end{array}$ & Exercise & $\begin{array}{l}\text { Caffeine } \\
\text { Dose }\end{array}$ & $\begin{array}{l}\text { Comparison } \\
\text { to best } \\
\text { practice? }\end{array}$ & Finding \\
\hline $\begin{array}{l}\text { Graham } \\
\text { and } \\
\text { Spriet } \\
\text { [39] }\end{array}$ & $\begin{array}{l}8 \text { well-trained } \\
\text { males }\end{array}$ & $\begin{array}{l}60 \text { minutes } \\
\text { pre- } \\
\text { exercise }\end{array}$ & $\begin{array}{l}\text { TTE run at } \\
85 \% \\
\text { VO2max }\end{array}$ & $\begin{array}{l}0 \\
\text { (placebo), } \\
3,6, \& 9 \\
\mathrm{mg} / \mathrm{kg}\end{array}$ & Yes & $\begin{array}{l}\text { Endurance was } \\
\text { equally enhanced } \\
\text { in both } 3 \text { and } 6 \\
\mathrm{mg} / \mathrm{kg} \text { caffeine } \\
\text { trials }\end{array}$ \\
\hline $\begin{array}{l}\text { Kovacs } \\
\text { et al. [40] }\end{array}$ & $\begin{array}{l}15 \text { well- } \\
\text { trained males }\end{array}$ & $\begin{array}{l}60 \% \text { of } \\
\text { solution } 60 \\
\text { minutes } \\
\text { pre- } \\
\text { exercise, } \\
\text { and } 20 \% \text { at } \\
\text { two time } \\
\text { points } \\
\text { within }\end{array}$ & $\begin{array}{l}\text { 1-hour } \\
\text { maximum } \\
\text { cycle }\end{array}$ & $\begin{array}{l}0 \\
\text { (Placebo), } \\
2.1,3.2, \\
4.5 \mathrm{mg} / \mathrm{kg}\end{array}$ & Yes & $\begin{array}{l}\text { Performance was } \\
\text { enhanced to the } \\
\text { greatest extent in } \\
4.5 \mathrm{mg} / \mathrm{kg} \text {, then } 3.2 \\
\mathrm{mg} / \mathrm{kg} \text {, then } 2.1 \\
\mathrm{mg} / \mathrm{kg} \text {. }\end{array}$ \\
\hline
\end{tabular}




\begin{tabular}{|c|c|c|c|c|c|c|}
\hline & & $\begin{array}{l}\text { exercise } \\
\text { trial. }\end{array}$ & & & & \\
\hline $\begin{array}{l}\text { Jenkins } \\
\text { et al. [44] }\end{array}$ & $\begin{array}{l}13 \text { trained } \\
\text { male cyclists }\end{array}$ & $\begin{array}{l}60 \text { minutes } \\
\text { pre- } \\
\text { exercise }\end{array}$ & $\begin{array}{l}15 \text { minutes } \\
\mathrm{VO} 2 \text { peak } \\
\text { performance } \\
\text { cycle }\end{array}$ & $\begin{array}{l}0 \\
\text { (placebo), } \\
1,2,3 \\
\mathrm{mg} / \mathrm{kg}\end{array}$ & No & $\begin{array}{l}\text { Compared to } \\
\text { placebo, only } 2 \\
\text { mg/kg significantly } \\
\text { enhanced } \\
\text { performance }\end{array}$ \\
\hline $\begin{array}{l}\text { Desbrow } \\
\text { et al. [43] }\end{array}$ & $\begin{array}{l}9 \text { trained male } \\
\text { cyclists }\end{array}$ & $\begin{array}{l}60 \text { minutes } \\
\text { pre- } \\
\text { exercise }\end{array}$ & $\begin{array}{l}120 \text { min } \\
\text { steady state } \\
\text { cycle, } \\
\text { followed by } \\
\text { TT. }\end{array}$ & $\begin{array}{l}0 \\
\text { (placebo), } \\
1.5,3 \\
\mathrm{mg} / \mathrm{kg}\end{array}$ & No & $\begin{array}{l}\text { No performance } \\
\text { enhancement with } \\
\text { caffeine }\end{array}$ \\
\hline $\begin{array}{l}\text { Irwin et } \\
\text { al. [50] }\end{array}$ & $\begin{array}{l}12 \text { trained } \\
\text { male cyclists }\end{array}$ & $\begin{array}{l}90 \text { minutes } \\
\text { pre- } \\
\text { exercise }\end{array}$ & Cycle TT & $\begin{array}{l}0 \\
\text { (placebo) } \\
\text { or } 3 \\
\mathrm{mg} / \mathrm{kg}\end{array}$ & No & $\begin{array}{l}\text { Caffeine enhances } \\
\text { performance } \\
\text { compared to } \\
\text { placebo }\end{array}$ \\
\hline $\begin{array}{l}\text { Desbrow } \\
\text { et al. [41] }\end{array}$ & $\begin{array}{l}16 \text { trained } \\
\text { cyclists }\end{array}$ & $\begin{array}{l}90 \text { minutes } \\
\text { pre- } \\
\text { exercise }\end{array}$ & $\begin{array}{l}60 \text { min cycle } \\
\text { at } 75 \% \text { peak } \\
\text { sustainable } \\
\text { power }\end{array}$ & $\begin{array}{l}0 \\
\text { (placebo), } \\
3,6 \\
\mathrm{mg} / \mathrm{kg}\end{array}$ & Yes & $\begin{array}{l}\text { No additional } \\
\text { benefit of } 6 \mathrm{mg} / \mathrm{kg} \\
\text { compared to } 3 \\
\mathrm{mg} / \mathrm{kg}\end{array}$ \\
\hline $\begin{array}{l}\text { Wiles et } \\
\text { al. [46] }\end{array}$ & $\begin{array}{l}34 \text { male } \\
\text { athletes }\end{array}$ & $\begin{array}{l}60 \text { minutes } \\
\text { pre- } \\
\text { exercise }\end{array}$ & $1500 \mathrm{~m}$ run & $\begin{array}{l}\text { mg from } \\
\text { coffee }(3 \mathrm{~g} \\
\text { total } \\
\text { coffee) }\end{array}$ & No & $\begin{array}{l}\text { Caffeine enhanced } \\
\text { performance. }\end{array}$ \\
\hline $\begin{array}{l}\text { Van } \\
\text { Nieuwen } \\
\text { hoven et } \\
\text { al. [47] }\end{array}$ & $\begin{array}{l}98 \text { well trained } \\
\text { male and } \\
\text { females }\end{array}$ & $\begin{array}{l}\text { At start, } \\
4.5,9 \text { and } \\
13.5 \mathrm{~km} \text { of } \\
\text { exercise } \\
\text { trial }\end{array}$ & $18 \mathrm{~km}$ run & $90 \mathrm{mg}$ & No & $\begin{array}{l}\text { No effect of } \\
\text { caffeine }\end{array}$ \\
\hline
\end{tabular}




\begin{tabular}{|c|c|c|c|c|c|c|}
\hline $\begin{array}{l}\text { Bridge \& } \\
\text { Jones } \\
{[48]}\end{array}$ & 8 male runners & $\begin{array}{l}60 \text { minutes } \\
\text { pre- } \\
\text { exercise }\end{array}$ & $8 \mathrm{~km}$ race & $\begin{array}{l}0 \\
\text { (Placebo), } \\
3 \mathrm{mg} / \mathrm{kg}, \\
\text { or no } \\
\text { suppleme } \\
\text { nt. }\end{array}$ & No & $\begin{array}{l}\text { Caffeine enhanced } \\
\text { performance. }\end{array}$ \\
\hline $\begin{array}{l}\text { Schubert } \\
\text { et al. [45] }\end{array}$ & 6 male runners & $\begin{array}{l}65 \text { minutes } \\
\text { pre- } \\
\text { exercise }\end{array}$ & $5 \mathrm{~km}$ run $\mathrm{TT}$ & $\begin{array}{l}0 \\
\text { (placebo), } \\
80 \mathrm{mg}, \\
140 \mathrm{mg} \text { ) }\end{array}$ & No & $\begin{array}{l}\text { No differences in } \\
\text { caffeine } \\
\text { consumption trials } \\
\text { when compared to } \\
\text { placebo. }\end{array}$ \\
\hline $\begin{array}{l}\text { Perez- } \\
\text { Lopez et } \\
\text { al. [52] }\end{array}$ & $\begin{array}{l}13 \text { elite female } \\
\text { volleyball } \\
\text { players }\end{array}$ & $\begin{array}{l}60 \text { minutes } \\
\text { pre- } \\
\text { exercise }\end{array}$ & $\begin{array}{l}\text { Volleyball } \\
\text { specific tests }\end{array}$ & $\begin{array}{l}0 \\
\text { (placebo) } \\
\text { and } 3 \\
\mathrm{mg} / \mathrm{kg}\end{array}$ & No & $\begin{array}{l}\text { Caffeine enhanced } \\
\text { performance. }\end{array}$ \\
\hline $\begin{array}{l}\text { Del Coso } \\
\text { et al. [51] }\end{array}$ & $\begin{array}{l}15 \text { male } \\
\text { volleyball } \\
\text { players }\end{array}$ & $\begin{array}{l}60 \text { minutes } \\
\text { pre- } \\
\text { exercise }\end{array}$ & $\begin{array}{l}\text { Volleyball } \\
\text { specific tests }\end{array}$ & $\begin{array}{l}0 \\
\text { (placebo) } \\
\text { and } 3 \\
\mathrm{mg} / \mathrm{kg}\end{array}$ & No & $\begin{array}{l}\text { Caffeine enhanced } \\
\text { performance. }\end{array}$ \\
\hline $\begin{array}{l}\text { Strecker } \\
\text { et al. [49] }\end{array}$ & $\begin{array}{l}10 \text { male tennis } \\
\text { players }\end{array}$ & $\begin{array}{l}90 \text { minutes } \\
\text { pre- } \\
\text { exercise }\end{array}$ & $\begin{array}{l}\text { Tennis skill } \\
\text { performance }\end{array}$ & $\begin{array}{l}0 \\
\text { (placebo) } \\
\text { and } 3 \\
\mathrm{mg} / \mathrm{kg}\end{array}$ & No & $\begin{array}{l}\text { Caffeine enhanced } \\
\text { performance. }\end{array}$ \\
\hline $\begin{array}{l}\text { Astorino } \\
\text { et al. [42] }\end{array}$ & $\begin{array}{l}15 \text { active } \\
\text { males }\end{array}$ & $\begin{array}{l}60 \text { minutes } \\
\text { pre- } \\
\text { exercise }\end{array}$ & $\begin{array}{l}40 \text { maximal } \\
\text { knee } \\
\text { extensions }\end{array}$ & $\begin{array}{l}0 \\
\text { (placebo), } \\
2,5 \\
\mathrm{mg} / \mathrm{kg}\end{array}$ & Yes & $\begin{array}{l}\text { Only the } 5 \mathrm{mg} / \mathrm{kg} \\
\text { dose enhanced } \\
\text { performance. }\end{array}$ \\
\hline $\begin{array}{l}\text { Talanian } \\
\& \text { Spriet } \\
{[57]}\end{array}$ & $\begin{array}{l}15 \text { cyclists } \\
(\mathrm{n}=4 \text { female })\end{array}$ & $\begin{array}{l}40(\sim 42 \% \\
\text { total }), 20 \\
(\sim 33 \%\end{array}$ & $\begin{array}{l}\text { Time to } \\
\text { completion } \\
\text { cycle }\end{array}$ & $\begin{array}{l}0 \\
\text { (placebo), }\end{array}$ & No & $\begin{array}{l}\text { Higher caffeine } \\
\text { dose enhanced } \\
\text { time-trial }\end{array}$ \\
\hline
\end{tabular}




\begin{tabular}{|c|c|c|c|c|c|c|}
\hline & & $\begin{array}{l}\text { total) and } 0 \\
(\sim 25 \%) \\
\text { minutes } \\
\text { pre-time } \\
\text { trial }\end{array}$ & $\begin{array}{l}\text { ergometer } \\
\text { test }\end{array}$ & $\begin{array}{l}\sim 1.5, \sim 2.9 \\
\mathrm{mg} / \mathrm{kg}\end{array}$ & & $\begin{array}{l}\text { performance to a } \\
\text { greater extent than } \\
\text { lower dose. }\end{array}$ \\
\hline $\begin{array}{l}\text { Tallis \& } \\
\text { Yavuz } \\
\text { [55] }\end{array}$ & $\begin{array}{l}10 \text { active } \\
\text { males }\end{array}$ & $\begin{array}{l}60 \text { minutes } \\
\text { pre- } \\
\text { exercise }\end{array}$ & $\begin{array}{l}\text { Isokinetic } \\
\text { concentric } \\
\text { and } \\
\text { eccentric } \\
\text { strength at } \\
60 \& 180 \\
\text { deg/s of } \\
\text { elbow and } \\
\text { knee flexors }\end{array}$ & $\begin{array}{l}0 \\
\text { (placebo), } \\
3 \text { and } 6 \\
\mathrm{mg} / \mathrm{kg}\end{array}$ & Yes & $\begin{array}{l}\text { No effect of } \\
\text { caffeine on elbow } \\
\text { flexor (concentric } \\
\text { and eccentric) or } \\
\text { knee (eccentric) } \\
\text { flexor strength. } \\
\text { Both caffeine } \\
\text { doses increased } \\
\text { concentric force in } \\
\text { knee extensors at } \\
180 \text { deg/s, with no } \\
\text { difference between } \\
\text { doses. Only the } \\
\text { higher (6 mg/kg) } \\
\text { dose enhanced } \\
\text { force during } \\
\text { repeated } \\
\text { contractions. }\end{array}$ \\
\hline $\begin{array}{l}\text { Turley et } \\
\text { al. [54] }\end{array}$ & $\begin{array}{l}26 \text { young (8- } \\
10 y) \text { boys }\end{array}$ & $\begin{array}{l}60 \text { minutes } \\
\text { pre- } \\
\text { exercise }\end{array}$ & $\begin{array}{l}\text { Hand grip } \\
\text { and Wingate } \\
\text { tests.. }\end{array}$ & $\begin{array}{l}0, \\
\text { (placebo), } \\
1,3 \text { and } 5 \\
\mathrm{mg} / \mathrm{kg}\end{array}$ & Yes & $\begin{array}{l}\text { Grip strength - } \\
\text { significantly higher } \\
\text { in } 3 \text { and } 5 \mathrm{mg} / \mathrm{kg} \\
\text { caffeine trials. } \\
\text { Wingate }-3 \mathrm{mg} / \mathrm{kg} \\
\text { produced greatest } \\
\text { peak power, whilst }\end{array}$ \\
\hline
\end{tabular}




\begin{tabular}{|c|c|c|c|c|c|c|}
\hline & & & & & & $\begin{array}{l}5 \mathrm{mg} / \mathrm{kg} \text { produced } \\
\text { greatest mean } \\
\text { power. }\end{array}$ \\
\hline $\begin{array}{l}\text { Arazi et } \\
\text { al. [53] }\end{array}$ & $\begin{array}{l}10 \text { female } \\
\text { karate athletes }\end{array}$ & $\begin{array}{l}60 \text { minutes } \\
\text { pre- } \\
\text { exercise }\end{array}$ & $\begin{array}{l}\text { 1RM leg } \\
\text { press, leg } \\
\text { press } \\
\text { repetitions to } \\
\text { failure, } \\
\text { vertical } \\
\text { jump, RAST } \\
\text { test. }\end{array}$ & $\begin{array}{l}0 \\
\text { (placebo), } \\
2 \text { and } 5 \\
\mathrm{mg} / \mathrm{kg}\end{array}$ & Yes & $\begin{array}{l}\text { No significant } \\
\text { difference in test } \\
\text { performance } \\
\text { between groups. }\end{array}$ \\
\hline $\begin{array}{l}\text { Sabol et } \\
\text { al [56] }\end{array}$ & $\begin{array}{l}20 \\
\text { recreationally } \\
\text { active males }\end{array}$ & $\begin{array}{l}60 \text { minutes } \\
\text { pre- } \\
\text { exercise }\end{array}$ & $\begin{array}{l}\text { Medicine } \\
\text { ball throw } \\
\text { and vertical } \\
\text { jump }\end{array}$ & $\begin{array}{l}0 \\
\text { (placebo), } \\
2,4, \text { and } 6 \\
\mathrm{mg} / \mathrm{kg}\end{array}$ & Yes & $\begin{array}{l}\text { No difference } \\
\text { between caffeine } \\
\text { doses in terms of } \\
\text { lower body } \\
\text { performance } \\
\text { enhancement. Only } \\
6 \text { mg/kg enhanced } \\
\text { upper body } \\
\text { performance. }\end{array}$ \\
\hline $\begin{array}{l}\text { Guest et } \\
\text { al [22] }\end{array}$ & $\begin{array}{l}101 \\
\text { competitive } \\
\text { males }\end{array}$ & $\begin{array}{l}45 \\
\text { minutes } \\
\text { pre- } \\
\text { exercise }\end{array}$ & $\begin{array}{l}\text { 10km cycle } \\
\text { ergometer } \\
\text { time trial }\end{array}$ & $\begin{array}{l}0 \\
\text { (placebo), } \\
2 \text { and } 4 \\
\mathrm{mg} / \mathrm{kg}\end{array}$ & Yes & $\begin{array}{l}\text { No difference in } \\
\text { performance } \\
\text { enhancement } \\
\text { between caffeine } \\
\text { doses; both } \\
\text { enhanced } \\
\text { performance } \\
\text { compared to } \\
\text { placebo. }\end{array}$ \\
\hline
\end{tabular}


161 Table 1 - A summary of studies examining the impact of low doses of pre-exercise caffeine on sports

162 performance. For the purposes of this table, a low dose of caffeine is defined as $3 \mathrm{mg} / \mathrm{kg}$ or less. (Adapted from

163 Spriet [24]; studies that did not utilise a pre-exercise caffeine dose, or those that only used a caffeine dose

164 greater than $3 \mathrm{mg} / \mathrm{kg}$, were excluded, and additional relevant papers published since that review have been added). 1RM; one repetition maximum. RAST; running-based anaerobic sprint test.

166

\section{A potential solution?}

168

This is not to suggest that these methodological shortcomings are the fault of researchers. Commonly, investigations are designed to explore phenomena tangentially bordering, but not directly targeting, this experimental question. However, based on our interpretation of the research, it is clear that, to decisively answer this question, additional trials that directly compare low caffeine doses with those falling into line with the currently accepted optimal dose (3-6 mg/kg), are required. Such research would remove much of the existing ambiguity permeating caffeine research. An equivalent approach is considered best-practice in the realm of medical drug development, where randomised controlled trials are designed to directly compare new drugs with the best currently available treatment as the optimal approach [58]. Accordingly, it is not sufficient to demonstrate that a new intervention is more effective than placebo, but that it produces better results than the currently accepted best treatment. ergogenic aid in sport [19]. Studies investigating the ergogenic effects of caffeinated gum on aerobic endurance performance are currently equivocal. As per a recent review [19]. two studies $[59,60]$ reported no ergogenic effect of caffeinated gum on aerobic endurance performance, whilst three studies [61-63] reported a positive

184 effect. An obvious distinction between these trials is the dose; the "no effect" findings occurred following a dose of $200 \mathrm{mg}$, whilst the positive effect trials employed a dose of $300 \mathrm{mg}$. If we assume an average subject mass of $\sim 80 \mathrm{~kg}$, then $200 \mathrm{mg}$ of caffeine would be classed as a low dose, and $300 \mathrm{mg}$ would fall within the recommended optimal threshold. Here, the inclusion of a trial utilising a currently accepted optimal caffeine dose in the $200 \mathrm{mg}$ studies would potentially resolve the current ambiguity. 
Additionally, there is contemporary debate regarding the impact of regular caffeine consumption on the

191 subsequent ergogenic effects of caffeine, with some studies finding a negative impact of habituation [32], whilst

192 others report none [64]. One potential outcome is that regular caffeine use requires a subsequently larger

193 caffeine dose to exert performance benefits [65]. As such, the dose of caffeine used in experimental trials

194 substantially influences study conclusions, particularly when exploring the effects of habitual use. Recently,

195 Evans and colleagues [66] explored the influence of caffeinated gum, supplying $200 \mathrm{mg}$ of caffeine, on repeated

196 sprint performance in team sport athletes. The initial finding was that caffeine did not confer any ergogenic

197 effects; however, further analysis demonstrated that habitual caffeine use modified the performance

198 enhancement seen following caffeine ingestion; in this case, very low habitual caffeine users $(<40 \mathrm{mg} / \mathrm{d}) \mathrm{did}$

199 exhibit ergogenic effects, whilst more moderate habitual users (>130 mg/d) did not. Such findings may be

200 interpreted as evidence that habitual use reduced caffeine's ergogenic effects. However, an obvious question

201 emerges; what if the dose of caffeine used was within the currently accepted guidelines, as opposed to <3

$202 \mathrm{mg} / \mathrm{kg}$ ? As this wasn't explored, the answer remains unclear. Again, this is not an attack on the authors, who were exploring a different research question, but it nevertheless underscores the point that increasingly robust conclusions could be inferred from caffeine research if the currently accepted optimal dose was included.

\section{How robust is the currently accepted optimal dose?}

207

For the purposes of this review, we have defined the currently accepted optimal dose of caffeine as between 3 and $6 \mathrm{mg} / \mathrm{kg}$. This figure is based on a number of different reviews and positions stands [14,23].

210 Furthermore, it is not suggested that there are any additional ergogenic effects associated with a dose above this

211 [25]. However, there is considerable inter-individual variation in the ergogenic effects of caffeine ingestion [68].

212 This phenomenon becomes apparent when caffeine studies report individual subject data. Jenkins et al. [44], for

213 example, examined the effects of lower caffeine doses $(1,2$, and $3 \mathrm{mg} / \mathrm{kg})$ compared to placebo on a 15 -minute

214 maximum cycle. Of the 13 subjects, one did not exhibit an ergogenic effect at any dose, whilst four found

215 caffeine ergogenic at every dose, but to different extents. Graham and Spriet [39] demonstrated that $9 \mathrm{mg} / \mathrm{kg}$ of

216 caffeine improved time-to-exhaustion in seven subjects, but with the percentage improvements compared

217 against the placebo trial varying from $105-250 \%$. Neither of these studies utilised the currently accepted optimal

218 caffeine dose, so whether the findings would have been replicated under those conditions remains unclear.

219 Nevertheless, the results serve to illustrate the extent of inter-individual responses to caffeine. Furthermore, 
some studies report no ergogenic effect of caffeine [67], again illustrating that the individual response to a standardised dose of caffeine is highly variable. The drivers of the variation of wide and varied, but can be grossly summarised as genetic, environmental (i.e. non-genetic), and epigenetic factors [68].

\subsection{Genetic}

Variation within CYP1A2, the gene encoding for cytochrome P450 1A2 - the enzyme responsible for 95\% of all caffeine metabolism [69]— has been shown to affect caffeine metabolization speed. Here, individuals with a $\mathrm{C}$ allele metabolise caffeine slower than AA genotypes [70]. Potentially, this single nucleotide polymorphism (SNP) might impact caffeine ergogenicity, with C allele carriers exhibiting lower [29] or no [22] ergogenic effects. However, these findings are currently tentative, with other studies reporting the opposite [71], or no effect [72] of this polymorphism on performance. The mechanism underpinning this reduced ergogenic effect in $\mathrm{C}$ allele carriers is currently unclear. Guest and colleagues [22] suggest that, because caffeine is a vasoconstrictor, slow metabolisers experience this vasoconstriction for a longer period of time, inhibiting the delivery of oxygen and nutrients to the working muscle. Conversely, Womack and colleagues [29] suggest that the downstream metabolites of caffeine (paraxanthine, theobromine, and theophylline) confer their own ergogenic effect; in this case, the presence of these metabolites would be lower in C allele carriers than AA genotypes at a given time point due to the slower metabolization of caffeine. As such, it's not clear whether caffeine has a reduced ergogenic, or even an ergolytic, effect in $\mathrm{C}$ allele carriers, or whether they need to ingest caffeine a greater amount of time before exercise [73]. Similarly, there is the potential that a SNP in ADORA2A, which encodes for a sub-type of adenosine receptor, may underpin some of the individual variation in response to caffeine, in terms of ergogenicity [30], anxiety [74], and sleep disturbances [75].

\subsection{Environmental}

Alongside these genetic drivers are environmental determinants of individual variation in the response to caffeine, which include age [35], training status [31], habitual caffeine use [32,65], diet [76], medication use [77], and personal belief as to whether caffeine enhances performance [36].

\subsection{Epigenetics}

Habitual caffeine use likely induces long-term epigenetic changes $[78,79]$, which may in turn affect future ergogenic effects, potentially by increasing caffeine metabolization speed [80]. For example, habitual 
caffeine use increases CYP1A2 activity [81], thereby increasing caffeine clearance, which may alter the expected ergogenic effects of caffeine ingestion. Additionally, long-term exposure to caffeine may alter its stimulatory effects, partly mediated by inhibition of genes affecting the adenosine pathway [82].

Accordingly, whilst caffeine is ergogenic, the currently accepted optimal caffeine dose may not be optimal for everyone [68]. Some individuals may benefit from lower doses of caffeine (discussed below), whilst others may need higher doses. Nevertheless, at present the abundance of evidence does suggest that, for most people, most of the time, a caffeine dose of between 3-6 mg/kg likely is sufficient to realise the optimum ergogenic effects. Indeed, Burke [83] suggested that the dose-response relationship of caffeine on performance appears to plateau at around $3 \mathrm{mg} / \mathrm{kg}$. As such, this dose may represent a target threshold to maximise caffeine's ergogenic effects, although higher doses are indeed ergogenic, and in some cases may be required, such as in habitual users [65]. Sensibly, the recommendations of 3-6 mg/kg should be taken as a starting point, from which individual experimentation can be used to refine pre-training and pre-competition caffeine strategies.

\section{When might lower doses of caffeine be more appropriate?}

The purpose of this article is not to discount the ergogenic potential of lower doses of caffeine; indeed, available evidence suggests that these lower doses can enhance performance [24]. Furthermore, the use of lower doses of caffeine may be preferential in certain situations. Higher doses of caffeine, for example, appear to be more likely to induce negative side-effects, such as anxiety [84] and sleep disturbances [85]. From a sporting perspective, both of these outcomes have the potential to negatively impact performance [86,87]. Furthermore, sleep disturbances following caffeine ingestion may reduce recovery from exercise and/or competition, and subsequently harm physical performance the following day [87]. In these cases, individual athletes need to make informed, strategic decisions negotiating the trade-off between the optimised ergogenic effects seen with higher

274 doses of caffeine against the potential for increased anxiety or compromised sleep. Here, the context is critical; arguably, the athlete would be more concerned with sleep disturbances if there is a high priority competitive bout in the proceeding few days, such as during the heats at the Olympic Games, as opposed to an Olympic Final, when no subsequent performance is required. Conversely, athletes predisposed to greater pre-competition anxiety may wish to consume less caffeine prior to important competitions than they would for lower level competitions and training, as caffeine may exacerbate this anxiety-promoting predisposition. 
282 caffeine. Preliminary evidence suggests, for example, that moderate doses of caffeine (4 mg/kg) are harmful to endurance performance in CYP1A2 genotypes [22]. However, a dose of $2 \mathrm{mg} / \mathrm{kg}$ showed no performance

284 decrement, suggesting that lower doses for these individuals may be more favourable than higher doses. Whilst further clarification is required, the potential for genetically-guided caffeine recommendations to be made, with certain genotypes potentially responding better to lower caffeine doses, remains a future possibility $[68,73]$. has been shown to negatively affect the ergogenic benefits of a caffeine dose [32,65], although this remains equivocal [64]. There is the potential that regular ingestion of caffeine increases the amount of caffeine required to realise the ergogenic effects, such that if an athlete habitually consumed $3 \mathrm{mg} / \mathrm{kg}$ of caffeine pre-training, they might require a caffeine dose closer to $6 \mathrm{mg} / \mathrm{kg}$ pre-competition [65]. This may increase the potential for adverse side effects, and, if the habitual dose increases over time, might take the athlete to a point in which further increases in dose don't restore the optimised ergogenic effect of caffeine. In this scenario, habitual use of lower caffeine doses $(\sim 3 \mathrm{mg} / \mathrm{kg})$ may facilitate an increased pre-competition dose, thereby allowing for both enhancement of regular training, along with competition performance.

\section{Conclusions}

In summary, the existing research is clear that low doses of caffeine are ergogenic [24]. However, to

301 derive more robust conclusions there is an evident need within these studies for a direct comparison with the

302 currently accepted optimal caffeine dose (>3 to $6 \mathrm{mg} / \mathrm{kg}$ ). The majority of studies that support the ergogenic benefits of low doses of caffeine do not compare these low doses to the caffeine doses more typically considered

304 to be ergogenic. As a result, whilst low doses of caffeine do offer a performance benefit, it's not clear that this performance benefit is greater than, or indeed equal to, that offered by caffeine doses between 3 and $6 \mathrm{mg} / \mathrm{kg}$.

306 The addition of a caffeine trial utilising $3-6 \mathrm{mg} / \mathrm{kg}$ of caffeine would therefore greatly aid in the interpretation of such findings, and so should be considered in future research. 
310 researchers to better critique the studies underpinning their caffeine strategies and recommendations. Moving

311 forward, we also recommend that caffeine researchers include a trial that utilizes the currently accepted optimal

312 dose of caffeine - even if this dose is not optimal for everyone - in order to enable more direct comparisons

313 between studies, and thereby enabling firmer conclusions to be made. Finally, as per our previous explorations

314 of caffeine use in sport $[65,68]$, we urge athletes and practitioners to experiment with different caffeine doses,

315 timing, and ingestion methods in order to uncover the strategies best suiting their unique genetic predispositions, environmental influences, and individual histories.

\section{Novelty Statement \& Practical Applications}

319 This critical review has demonstrated that, whilst lower doses ( $\leq 3 \mathrm{mg} / \mathrm{kg}$ ) of caffeine have the potential to be ergogenic, it's not clear whether such doses are as ergogenic as higher doses. The main cause of this uncertainty is due to a lack of trials directly comparing low and high doses of caffeine. As such, athletes, coaches and practitioners looking to utilise caffeine as a means to enhance performance would be best placed to experiment with various different caffeine doses in order to determine the optimal dose to enhance their performance, given their own unique biology, history, and performance requirements.

\section{Compliance with Ethical Standards}

327 Funding

328 No sources of funding were used to assist in the preparation of this article.

\section{References:}

1. Desbrow B, Leveritt M. (2006). Awareness and use of caffeine by athletes competing at the 2005 Ironman Triathlon World Championships. Int J Sport Nutr Exerc Metab 16(5):545-58.

2. Del Coso J, Muñoz G, Muñoz-Guerra J. (2011). Prevalence of caffeine use in elite athletes following its removal from the World Anti-Doping Agency list of banned substances. Appl Physiol Nutr Metab 36(4):555-61

3. Keisler BD, Armsey TD. (2006). Caffeine as an ergogenic aid. Curr Sports Med Rep 5(4):215-9. 
4. Foskett A, Ali A, Gant N. (2009). Caffeine enhances cognitive function and skill performance during simulated soccer activity. Int J Sport Nutr Exerc Metab 19(4):410-23.

5. Glaister M, Howatson G, Abraham CS, et al. (2008). Caffeine supplementation and multiple sprint running performance. Med Sci Sports Exerc 40(10):1835-40.

6. Da Silva VL, Messias FR, Zanchi NE, et al. (2015). Effects of acute caffeine ingestion on resistance training performance and perceptual responses during repeated sets to failure. J Sports Med Phys Fitness 55(5):383-9.

7. Grgic J, Mikulic P. (2017). Caffeine ingestion acutely enhances muscular strength and power but not muscular endurance in resistance-trained men. Eur J Sport Sci 17(8):1029-1036.

8. Grgic J. (2018). Caffeine ingestion enhances Wingate performance: a meta-analysis. Eur J Sport Sci 18(2):219-225.

9. Rivers WH, Webber HN. (1907). The action of caffeine on the capacity for muscular work. J Physiol $36(1): 33$

10. Biaggioni IT, Paul SU, Puckett AN, et al. (1991). Caffeine and theophylline as adenosine receptor antagonists in humans. J Pharmacol Exp Ther 258(2):588-93.

11. Ribeiro JA, Sebastiao AM. (2010). Caffeine and adenosine. J Alzheimers Dis 20(S1):3-15.

12. Fredholm BB. (1995). Adenosine, adenosine receptors and the actions of caffeine. Pharmacol Toxicol 76(2):93-101.

13. Kalmar JM. (2005). The influence of caffeine on voluntary muscle activation. Med Sci Sports Exerc 37(12):2113-9.

14. Graham TE. (2001). Caffeine and exercise: Metabolism, endurance and performance. Sports Med 31(11):785-807.

15. Cruz RS, de Aguiar RA, Turnes T, et al. (2015). Caffeine affects time to exhaustion and substrate oxidation during cycling at maximal lactate steady state. Nutrients 7(7):5254-64.

16. Sökmen B, Armstrong LE, Kraemer WJ, et al. (2008). Caffeine use in sports: considerations for the athlete. J Strength Cond Res 22(3):978-86.

17. Doherty M, Smith PM, Hughes MG, et al. (2004). Caffeine lowers perceptual response and increases power output during high-intensity cycling. J Sports Sci 1;22(7):637-43.

18. Gonglach AR, Ade CJ, Bemben MG, et al. (2016). Muscle pain as a regulator of cycling intensity: effect of caffeine ingestion. Med Sci Sports Exerc 48(2):287-96 
19. Wickham KA, Spriet LL. (2018). Administration of Caffeine in Alternate Forms. Sports Med 48(S1):79-91.

20. Gam S, Guelfi KJ, Fournier PA. (2016). New insights into enhancing maximal exercise performance through the use of a bitter tastant. Sports Med 46(10):1385-90.

21. Beaven CM, Maulder P, Pooley A, et al. (2013). Effects of caffeine and carbohydrate mouth rinses on repeated sprint performance. Appl Physiol Nutr Metab 38(6):633-7.

22. Guest N, Corey P, Vescovi J, et al. (2018). Caffeine, CYP1A2 genotype, and endurance performance in athletes. Med Sci Sports Exerc 50(8):1570-8.

23. Goldstein ER, Ziegenfuss T, Kalman D, et al. (2010). International society of sports nutrition position stand: caffeine and performance. J Int Soc Sports Nutr 7(1):5.

24. Spriet LL. (2014). Exercise and sport performance with low doses of caffeine. Sports Med 44(2):17584.

25. Pasman WJ, Van Baak MA, Jeukendrup AE, et al. (1995). The effect of different dosages of caffeine on endurance performance time. Int J Sports Med 16(4):225-30.

26. Cox GR, Desbrow B, Montgomery PG, et al. (2002). Effect of different protocols of caffeine intake on metabolism and endurance performance. J Appl Physiol 93(3):990-9.

27. Souza DB, Del Coso J, Casonatto J, et al. (2017). Acute effects of caffeine-containing energy drinks on physical performance: a systematic review and meta-analysis. Eur J Nutr 56(1):13-27.

28. Ganio MS, Klau JF, Casa DJ, et al. (2009). Effect of caffeine on sport-specific endurance performance: a systematic review. J Strength Cond Res 23(1):315-24.

29. Womack CJ, Saunders MJ, Bechtel MK, et al. (2012). The influence of a CYP1A2 polymorphism on the ergogenic effects of caffeine. J Int Soc Sports Nutr 9(1):7.

30. Loy BD, O'Connor PJ, Lindheimer JB, et al. (2015). Caffeine is ergogenic for adenosine A2A receptor gene (ADORA2A) T allele homozygotes: a pilot study. J Caffeine Res 5(2):73-81.

31. Collomp K, Ahmaidi S, Chatard JC, et al. (1992). Benefits of caffeine ingestion on sprint performance in trained and untrained swimmers. Eur J Appl Physiol Occup Physiol 64(4):377-80.

32. Beaumont R, Cordery P, Funnell M, et al. (2017). Chronic ingestion of a low dose of caffeine induces tolerance to the performance benefits of caffeine. J Sports Sci 35(19):1920-1927. strength and muscular endurance. Comparative Exercise Physiology 11(2):89-94. 
34. Hodgson AB, Randell RK, Jeukendrup AE. (2013). The metabolic and performance effects of caffeine compared to coffee during endurance exercise. PLoS One 8(4):e59561.

35. Tallis J, James RS, Cox VM, et al. (2017). Is the ergogenicity of caffeine affected by increasing age? The direct effect of a physiological concentration of caffeine on the power output of maximally stimulated edl and diaphragm muscle isolated from the mouse. J Nutr Health Aging 21(4):1-9

36. Saunders B, Oliveira LF, Silva RP, et al. (2017). Placebo in sports nutrition: a proof- of- principle study involving caffeine supplementation. Scand J Med Sci Sports 27(11):1240-1247 doi: 10.1111/sms.12793.

37. Davis JK, Green JM. (2009). Caffeine and anaerobic performance. Sports Med 39(10):813-32.

38. Mora-Rodríguez R, Pallarés JG, López-Gullón JM, et al. (2015). Improvements on neuromuscular performance with caffeine ingestion depend on the time-of-day. J Sci Med Sport 18(3):338-42.

39. Graham TE, Spriet LL. (1995). Metabolic, catecholamine, and exercise performance responses to various doses of caffeine. J Appl Physiol 78(3):867-74

40. Kovacs EM, Stegen JH, Brouns F. (1998). Effect of caffeinated drinks on substrate metabolism, caffeine excretion, and performance. J Appl Physiol 85(2):709-15.

41. Desbrow B, Biddulph C, Devlin B, et al. (2012). The effects of different doses of caffeine on endurance cycling time trial performance. J Sports Sci 30(2):115-20.

42. Astorino TA, Terzi MN, Roberson DW, et al. (2010). Effect of two doses of caffeine on muscular function during isokinetic exercise. Med Sci Sports Exerc 42(12):2205-10.

43. Desbrow B, Barrett CM, Minahan CL, et al. (2009). Caffeine, cycling performance, and exogenous CHO oxidation: a dose-response study. Med Sci Sports Exerc 41(9):1744-51. performance. Int J Sport Nutr Exerc Metab 18(3):328-42.

45. Schubert MM, Astorino TA. (2013). The effects of caffeinated "energy shots" on time trial performance. Nutrients 5(6):2062-75.

46. Wiles JD, Bird SR, Hopkins J, et al. (1992). Effect of caffeinated coffee on running speed, respiratory factors, blood lactate and perceived exertion during 1500-m treadmill running. Br J Sports Med 26(2):116-20. GI complaints and performance during an 18-km run. Int J Sports Med 26(04):281-5. 
48. Bridge CA, Jones MA. (2006). The effect of caffeine ingestion on $8 \mathrm{~km}$ run performance in a field setting. J Sports Sci 24(4):433-9.

49. Strecker E, Foster B, Taylor K, et al. (2006). The effect of caffeine ingestion on tennis skill performance. Med Sci Sports Exerc 38(5):S175.

50. Irwin C, Desbrow B, Ellis A, et al. (2011). Caffeine withdrawal and high-intensity endurance cycling performance. J Sports Sci 29(5):509-15.

51. Del Coso J, Pérez-López A, Abian-Vicen J, et al. (2014). Enhancing physical performance in male volleyball players with a caffeine-containing energy drink. Int J Sports Physiol Perform 9(6):1013-8.

52. Perez-Lopez A, Salinero JJ, Abian-Vicen J, et al. (2015). Caffeinated energy drinks improve volleyball performance in elite female players. Med Sci Sports Exerc 47(4):850-6.

53. Arazi H, Hoseinihaji M, Eghbali E. (2016). The effects of different doses of caffeine on performance, rating of perceived exertion and pain perception in teenagers female karate athletes. Braz J Pharm Sci 52(4):685-92.

54. Turley KR, Eusse PA, Thomas MM, et al. (2015). Effects of different doses of caffeine on anaerobic exercise in boys. Pediatr Exerc Sci 27(1):50-6.

55. Tallis J, Yavuz HC. (2017). The effects of low and moderate doses of caffeine supplementation on upper and lower body maximal voluntary concentric and eccentric muscle force. Appl Physiol Nutr Metab 43(3):274-81.

56. Sabol F, Grgic J, Mikulic P. (2019) The effects of three different doses of caffeine on jumping and throwing performance: a randomized, double-blind, crossover study. Int J Sports Physiol Perform doi: 10.1123/ijspp.2018-0884

57. Talanian JL, Spriet, LL. (2016). Low and moderate doses of caffeine late in exercise improve performance in trained cyclists. Appl Physiol Nutr Metab 41(8):850-5.

58. Henry D, Hill S. (1995). Comparing treatments. BMJ 310(6990):1279.

59. Ryan EJ, Kim CH, Muller MD, et al. (2012). Low-dose caffeine administered in chewing gum does not chewing gum: independent and combined effects on endurance cycling performance. Int J Sports Physiol Perform 11(2):164-71. 
61. Ryan EJ, Kim CH, Fickes EJ, et al. (2013). Caffeine gum and cycling performance: a timing study. J Strength Cond Res 27(1):259-64.

62. Lane SC, Hawley JA., Desbrow B, et al. (2013). Single and combined effects of beetroot juice and caffeine supplementation on cycling time trial performance. Appl Physiol Nutr Metab 39(9):1050-7.

63. Paton C, Costa V, Guglielmo L. (2015). Effects of caffeine chewing gum on race performance and physiology in male and female cyclists. J Sports Sci 33(10):1076-83.

64. Gonçalves L, de Salles Painelli V, Yamaguchi G, et al. (2017). Dispelling the myth that habitual caffeine consumption influences the performance response to acute caffeine supplementation. J Appl Physiol 123(1):213

65. Pickering C, Kiely J. (2018). What should we do about habitual caffeine use in athletes? Sports Med https://doi.org/10.1007/s40279-018-0980-7

66. Evans M, Tierney P, Gray N, et al. (2018). Acute ingestion of caffeinated chewing gum improves repeated sprint performance of team sports athletes with low habitual caffeine consumption. Int J Sport Nutr Exerc Metab 28(3):221-227. doi: 10.1123/ijsnem.2017-0217

67. Skinner TL, Jenkins DG, Coombes JS, et al. (2010). Dose response of caffeine on 2000-m rowing performance. Med Sci Sports Exerc 42(3):571-6.

68. Pickering C, Kiely J. (2018). Are the current guidelines on caffeine use in sport optimal for everyone? Inter-individual variation in caffeine ergogenicity, and a move towards personalised sports nutrition. Sports Med 48(1):7-16.

69. Gu L, Gonzalez FJ, Kalow W, et al. (1992). Biotransformation of caffeine, paraxanthine, theobromine and theophylline by cDNA-expressed human CYP1A2 and CYP2E1. Pharmacogenetics 2(2):73-7.

70. Sachse C, Brockmöller J, Bauer S, et al. (1999). Functional significance of a C $\rightarrow$ A polymorphism in intron 1 of the cytochrome P450 CYP1A2 gene tested with caffeine. Br J Clin Pharmacol 47(4):445-9.

71. Pataky MW, Womack CJ, Saunders MJ, et al. (2015). Caffeine and 3- km cycling performance: Effects of mouth rinsing, genotype, and time of day. Scand J Med Sci Sports 26(6):613-9.

72. Salinero JJ, Lara B, Ruiz-Vicente D, et al. (2017). CYP1A2 genotype variations do not modify the benefits and drawbacks of caffeine during exercise: a pilot study. Nutrients 9(3):269.

73. Pickering C. (2018). Caffeine, CYP1A2 genotype, and sports performance: is timing important? Ir J Med Sci doi: 10.1007/s11845-018-1811-4 
74. Alsene K, Deckert J, Sand P, et al. (2003). Association between A2a receptor gene polymorphisms and caffeine-induced anxiety. Neuropsychopharmacology 28(9):1694.

75. Retey JV, Adam M, Khatami R, et al. (2007). A genetic variation in the adenosine A2A receptor gene (ADORA2A) contributes to individual sensitivity to caffeine effects on sleep. Clin Pharmacol Ther 81(5):692-8.

76. Lampe JW, King IB, Li S, et al. (2000). Brassica vegetables increase and apiaceous vegetables decrease cytochrome P450 1A2 activity in humans: changes in caffeine metabolite ratios in response to controlled vegetable diets. Carcinogenesis 21(6):1157-62.

77. Abernethy DR, Todd EL. (1985). Impairment of caffeine clearance by chronic use of low-dose oestrogen-containing oral contraceptives. Eur J Clin Pharmacol 28(4):425-8.

78. Ping J, Wang JF, Liu L, et al. (2014). Prenatal caffeine ingestion induces aberrant DNA methylation and histone acetylation of steroidogenic factor 1 and inhibits fetal adrenal steroidogenesis. Toxicology 321:53-61

79. Wendler C, Poulsen R, Fang X. (2014). Caffeine induces both short-term and long-term effects on gene expression and DNA methylation in the mouse heart. FASEB 28(S1):542-3.

80. Jin B, Park DW, Nam KW, et al. (2004). CpG methylation of the mouse CYP1A2 promoter. Toxicol Letters 152(1):11-8.

81. Djordjevic N, Ghotbi R, Bertilsson L, et al. (2008). Induction of CYP1A2 by heavy coffee consumption in Serbs and Swedes. Eur J Clin Pharmacol 64(4):381-5.

82. Marques S, Batalha VL, Lopes LV, et al. (2011). Modulating Alzheimer's disease through caffeine: a putative link to epigenetics. J Alzheimers Dis 24(S2):161-71.

83. Burke LM. (2008). Caffeine and sports performance. Appl Physiol Nutr Metab 33(6):1319-34.

84. Evans SM, Griffiths RR. (1991). Dose-related caffeine discrimination in normal volunteers: individual differences in subjective effects and self-reported cues. Behav Pharmacol 2(4\&5):345-356.

85. Karacan I, Thornby JI, Anch AM, et al. (1976). Dose- related sleep disturbances induced by coffee and caffeine. Clin Pharmacol Ther 20(6):682-9.

86. Woodman T, Hardy L. (2003). The relative impact of cognitive anxiety and self-confidence upon sport performance: A meta-analysis. J Sports Sci 21(6):443-57.

87. Reilly T, Edwards B. (2007). Altered sleep-wake cycles and physical performance in athletes. Physiol Behav 90(2-3):274-84. 
